R. Tamburo, A. Cois, D. Shelton, G. Stetten, "Medial Node Correspondences for Automatic Non-Rigid Registration," Second International Workshop on Biomedical Image Registration, June, 2003, Philadelphia. Lecture Notes in Computer Science, vol. 2717, pp. 337-348.

\title{
Medial Node Correspondences Towards Automated Registration
}

\author{
Robert Tamburo $^{1 \dagger}$, Aaron Cois ${ }^{1 \ddagger}$, Damion Shelton ${ }^{2 \star}$, George Stetten ${ }^{1,2 \pm}$ \\ ${ }^{1}$ Department of Bioengineering, University of Pittsburgh \\ rjtst21@pitt.edu ${ }^{\dagger}$, cacst11@pitt.edu \\ ${ }^{2}$ Robotics Institute, Carnegie Mellon University \\ beowulf@cs.cmu.edu ${ }^{\star}$,george@stetten.com ${ }^{ \pm}$
}

\begin{abstract}
Many modern forms of segmentation and registration require manual input making it a tedious and time-consuming process. There have been some successes with automating these methods, but these tend to be unreliable because of inherent variations in anatomical shapes and image quality. It is toward this goal that we have developed an automated method of generating landmarks for registration that will not require supervision or manual initialization. We have chosen medial based image features because they have proven robust against image noise and shape variation, and provide the rotationally invariant properties of dimensionality and scale, which can be used by a unary metric. We introduce a new metric for comparing the geometric relationships between medial features, which overcomes problems introduced by symmetry within a medial feature. With these metrics, we are able to find correspondences between pairs and triplets of features in the two images. We demonstrate these methods on three different datasets. It is envisioned that this system will become the basis for generating medial node models that can be registered between two images."
\end{abstract}

\section{Introduction}

For over a century, medical imaging has permitted doctors and other healthcare workers to look beneath the skin's surface to obtain critical diagnostic information. In recent years, computational techniques have been developed to assist clinicians with the analysis of medical images. These techniques include methods for segmentation and registration. Segmentation facilitates the extraction of interesting shapes and the estimation of shape parameters for characterizing disease by classifying regions in the image. Registration is the process of aligning two or more images, valuable for superimposing structures from different imaging modalities and conducting comparative studies of anatomy across a population.

Many current forms of registration require manual input, making it a tedious and time-consuming process. There have been some successes with automated methods, but these tend to be unreliable because of inherent anatomical variation, image noise,

\footnotetext{
* This research was supported by a contract with the National Library of Medicine as part of the Insight Toolkit (ITK) software consortium. All software was developed and written with ITK.
} 
and discontinuous object boundaries ${ }^{[1-3]}$. Our approach is to automatically generate landmarks based on medial features, and we are able to find correspondences between these landmarks in two images. It has previously been shown that correspondences between image features can be found based on geometric constraints ${ }^{[4]}$. Our method is based on geometric constraints using cliques of medial features formed from rotationally invariant metrics. In this paper, we will show that similar objects in two images can be identified by sets of medial features and by the geometric relationships within these sets.

\section{Background}

A brief description of medialness, core atoms, and our methods to extract medial features is included in this section. For a more detailed description see $[5,6]$. The medial axis on an object, as defined by Blum for binary images, is the locus of centers of circles that are at least bi-tangent to the object boundary and fit entirely within the object ${ }^{[7]}$. Pizer et al. developed a measure called medialness to extend the medial axis to a medial manifold for gray-level images, where a distinct boundary is replaced by a measure called boundariness ${ }^{[8]}$. Many techniques for measuring medialness (including our own) link the aperture of the boundariness measurement to the radius of the medial manifold. A ridge of medialness is called a core. Methods using medialness have proven robust against image noise and shape variation $[5,6,9,10]$. There are several ways to locate the core. We use core atoms because they are efficient, requiring only a single application to detect an object ${ }^{[5]}$.

\subsection{Core Atoms}

The first step in the formation of core atoms is the collection of boundary points. Any boundary detection algorithm can be used so long as it delivers gradient magnitude and orientation. We have chosen to use a Differences of Offset Gaussian (DOOG) gradient detector $^{[11]}$ whose implementation is described in [12]. A core atom is formed from a pair of boundary points that meet the following requirements:

1. The distance between the boundary points is within a specified range. This distance is termed the scale, or diameter, of the resulting core atom and is defined as

$$
\left\|\mathbf{s}_{1,2}\right\|=\left\|\mathbf{b}_{2}-\mathbf{b}_{1}\right\|
$$

where $s_{\min } \leq\left\|\mathbf{s}_{1,2}\right\| \leq s_{\max }$ and the expected diameter is between $s_{\min }$ and $s_{\max }$. The vector $\mathbf{s}_{1,2}$ indicates the direction from the first boundary point location $\mathbf{b}_{1}$ to the second boundary point location $\mathbf{b}_{2}$ and the core atom is said to be "located" at the midpoint between the two boundary points. Figure 1 illustrates a core atom created across an object of intensity $I_{1}$ against a background of intensity $I_{2}$. 
2. The face-to-faceness, as defined in Eq. 2, of the constituent boundaries must be sufficiently close to 1 , while still allowing some variation in the relative orientations of those boundaries.

$$
F\left(\mathbf{b}_{1}, \mathbf{b}_{2}\right)=\left|\left(\frac{\mathbf{s}_{1,2}}{\left\|\mathbf{s}_{1,2}\right\|} \cdot \hat{\mathbf{n}}_{1}\right)\left(\frac{\mathbf{s}_{2,1}}{\left\|\mathbf{s}_{2,1}\right\|} \cdot \hat{\mathbf{n}}_{2}\right)\right|,
$$

where $\hat{\mathbf{n}}_{i}(i=1,2)$ is the orientation of the $i^{\text {th }}$ boundary point (the "^" notation denotes a normalized vector).

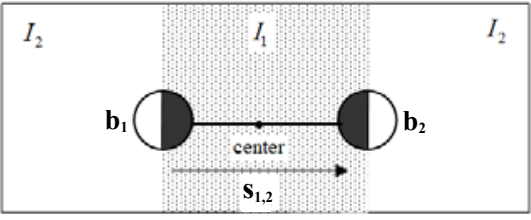

Fig. 1. A core atom consists of two boundary points separated by distance $\left\|\mathbf{s}_{1,2}\right\|$ with a center point midway between the boundary locations.

\subsection{Extracting Medial Properties with Core Atoms}

Populations of core atoms can be analyzed for a measure of medial dimensionality. For 3D data, there are three basic core atom configurations; "koosh-ball," "spokesof-a-wheel," and "bed-of-nails." The lower half of Fig. 2 illustrates these configurations, where line segments represent core atoms. Above each core atom configuration is the corresponding shape shown in dark gray and the core in light gray.

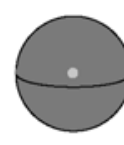

sphere

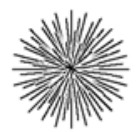

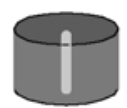

cylinder

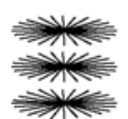

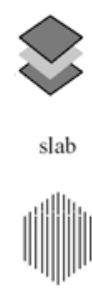

Fig. 2. Top row shows basic shapes in dark gray with corresponding cores in light gray. Below these are shown the corresponding basic core atom configurations (from left to right): "kooshball," "spokes-of-a-wheel," and "bed-of-nails" (with core atoms shown as line segments).

For a given population of core atoms, the orientation vectors can be statistically processed using eigenanalysis of their covariance matrix, to yield a measure of dimensionality and an overall estimate of orientation. The eigenvalues $\lambda_{1}<\lambda_{2}<\lambda_{3}$ define the dimensionality of the core and the corresponding eigenvectors $\hat{\mathbf{a}}_{1}, \hat{\mathbf{a}}_{2}$, and 
$\hat{\mathbf{a}}_{3}$ define a coordinate system specific to the orientation of the core atom population. The eigenvectors are ordered such that $\hat{\mathbf{a}}_{1}$ is most orthogonal to the population of core atom vectors and $\hat{\mathbf{a}}_{3}$ is least orthogonal. The eigenvalues are greater than or equal to 0 and sum to 1 . A value of 0 indicates that the corresponding eigenvector is completely orthogonal to every core atom, indicating a direction along the core. This is true for $\hat{\mathbf{a}}_{1}$ in a cylinder and $\hat{\mathbf{a}}_{1}$ and $\hat{\mathbf{a}}_{2}$ in a slab. For the sphere, whose core is a point, no eigenvectors are orthogonal to every core atom. Since the eigenvalues sum to 1 , they can be viewed as a system with only 2 independent variables, which define a triangular domain called the lambda triangle (shown with results in Figs 6). The vertices the lambda triangle represent the three basic medial shapes (sphere, cylinder, and slab). All possible sets of eigenvalues are bounded by the lambda triangle.

We sample core atoms first on a regular spatial grid. The core atom centers form a cloud around the true core, because of tolerance in the face-to-faceness. Medial properties measured in samples that are displaced from the true core are prone to distortion. This may cause a misclassification of local dimensionality. Further clustering of core atoms in adjacent samples overcomes this problem, using methods described by Stetten ${ }^{[5]}$. Each core atom population clustered in this manner is considered a single medial node. We define the location of a medial node as the center of mass of the corresponding core atom population, and the scale of the medial node as the average diameter of that population.

\section{Methods}

Finding correspondences between medial nodes in two images begins with the unary metric. Let $\Psi_{\alpha}$ be the set of all medial nodes in an $N$-dimensional image $\alpha$. For our applications thus far $N=3$, but the approach applies to any $N>1$. The images are designated $\mathrm{A}$ and $\mathrm{B}$, namely $\alpha=A$ or $B$.

\subsection{Medial Node Unary Metric}

Given that core atoms have been constructed and clustered into nodes, and the dimensionality, scale, and orientation of each node has been determined, the unary metric can be calculated between each node in $\Psi_{A}$ and each node in $\Psi_{B}$. The unary metric quantifies the similarity between a pair of medial nodes, independent of orientation, by their medial dimensionality and scale. Recall that each medial node has eigenvalues $\lambda_{i}, i=1,2, \ldots N$ and a scale $\sigma$. From these quantities, the unary metric $\mu_{j, k}$ of node $j$ in image A and node $k$ in image B is calculated by

$$
\mu_{j, k}=\left(\sum_{i=1}^{N-1}\left(\lambda_{j, i}-\lambda_{k, i}\right)^{2}\right)\left(\left|\frac{\sigma_{j}-\sigma_{k}}{\sigma_{j}+\sigma_{k}}\right|\right),
$$


where $\lambda_{j, i}$ and $\lambda_{k, i}$ are the $i^{\text {th }}$ eigenvalues for node $j$ and $k$ respectively, and $\sigma_{j}$ and $\sigma_{k}$ are the corresponding scales. Only the first $N-1$ eigenvalues are needed because only that many are independent.

The unary metric has a value between 0 and 1, where 0 indicates identical nodes. All of the metrics found between $\Psi_{A}$ and $\Psi_{B}$ are stored in a unary correspondence matrix. This matrix is $M_{A} x M_{B}$ in size, where $M_{A}$ and $M_{B}$ are the numbers of medial nodes in image A and B respectively. The unary correspondence matrix thus stores the similarity between every node in image A and every node in image B.

\subsection{Medial Node Signature Metric}

In this section, we will define the signature metric, to be used in conjunction with the unary metric. We will use the signature metric to find correspondences between similar pairs of medial nodes, also called 2-cliques, in two images. This metric produces two unique signatures for each pair of medial nodes in an image, one signature from the perspective of each node in the pair. The signature metric is used to find corresponding pairs in another image.

Consider a pair of medial nodes in an image. Given the eigenvectors $\hat{\mathbf{a}}_{i}, i=1,2, \ldots N$ of node 1 , the relative location of node 2 can be expressed in the coordinate system of node 1 as

$$
d_{j}=\sum_{i=1}^{N} v_{i}\left(\hat{\mathrm{a}}_{\mathrm{j}}\right)_{i},
$$

where $\left(\hat{\mathrm{a}}_{\mathrm{j}}\right)_{i}$ is the $i^{\text {th }}$ component of eigenvector $j$ and $\mathbf{v}$ is the displacement from node 1 to node 2 in the global coordinate system. We further define $\hat{\mathbf{d}}$ as the normalized vector

$$
\hat{\mathbf{d}}=\frac{\mathbf{d}}{\|\mathbf{d}\|},
$$

which expresses the direction to node 2 from node 1 in the coordinate system defined by the eigenvectors of node 1 . We will refer to the square of the normalized component $\left(\hat{d}_{i}\right)^{2}$ as the $\mathrm{i}^{\text {th }}$ Normalized Component Squared (NCS) of $\hat{\mathbf{d}}$.

A metric to express the similarity between node pairs should ignore symmetry in node 1 in terms of the location of node 2 (and vice versa). Such symmetry results only when two of node 1's eigenvalues are approximately equal. The signature for node 1 is defined as the cumulative NCS function, with the NCS for each component of $\hat{\mathbf{d}}$ (along a given eigenvector $\hat{\mathbf{a}}_{i}$ ) being added at its corresponding eigenvalue,

$$
s(\lambda)=\sum_{i=1}^{N}\left(\hat{d}_{i}\right)^{2} u\left(\lambda_{i}\right),
$$


where $u(\cdot)$ is the unit step function.

Since the eigenvalues are positive, $s(\lambda)=0$, for $\lambda<0$. In effect, $s(\lambda)$ is a series of steps at each of the eigenvalues, the height of each step being equal to the corresponding NCS. If two or more eigenvalues are equal, their steps superimpose into a single NCS in the degenerate subspace defined by the corresponding eigenvectors. By the Pythagorean Theorem, the total step size in such a case is independent of arbitrary rotation within the degenerate subspace. Also by the Pythagorean Theorem, the total of all the steps is $s(1)=1$.
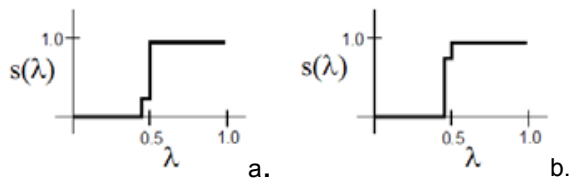

Fig. 3. a) Signature of cylindrical node 1 paired with node 2 at the "equator" of node 1's coordinate system. b) Another node pair also with an "equatorial" node 2, but a different orientation of node 1's eigenvectors within the degenerate subspace.

Typical signatures are shown in Fig. 3 for a pair of nodes where node 1 is almost perfectly cylindrical (recall that $\lambda_{1}=0, \lambda_{2}=0.5$, and $\lambda_{3}=0.5$ for a perfectly cylindrical node). In each pair, the second node is at the "equator" of the first node's coordinate system, but there is arbitrary rotation of the eigenvectors within the degenerate subspace around the axis of the cylinder. The two signatures vary along a very short segment of the $\lambda$-axis, and so are very similar. In Figure 4 , we show signatures from the same cylindrical node 1 , but this time with node 2 located not at the equator, but at $45^{\circ}$ "latitude."
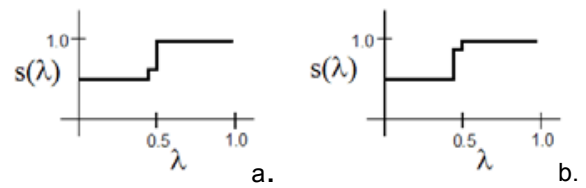

Fig. 4. a) Signature of cylindrical node 1 paired with node 2 at $45^{\circ}$ "latitude" of node 1 's coordinate system. b) Another equivalent node pair, as in Fig 3 b.

Let us look in more detail at the claim that a signature is independent of the global orientation of a node pair. A medial node will produce eigenvectors that depend on orientation with respect to the cardinal coordinate system. However, the eigenvalues are independent of that orientation. When two or more eigenvalues are equal, a degenerate subspace exists that may be expressed by matrix A whose columns are the corresponding eigenvectors $\mathbf{A}=\left[\begin{array}{lll}\hat{\mathbf{a}}_{\mathbf{i}} & \hat{\mathbf{a}}_{\mathbf{j}} & \ldots\end{array}\right]$.

Rotating a node pair within the subspace defined by the degenerate eigenvectors of node 1 will redefine the eigenvectors as $\mathbf{E}=\left[\hat{\mathbf{e}}_{\mathbf{i}} \hat{\mathbf{e}}_{\mathbf{j}} \ldots\right]=\mathbf{R A}$, where $\mathbf{R}$ is a pure rota- 
tion matrix. The cumulative NCS between node 1 and node 2 will remain unchanged. We demonstrate this for the case of two eigenvalues being equal. Rotation within the degenerate subspace is represented by

$$
\begin{aligned}
& \hat{\mathbf{e}}_{i}=\alpha \hat{\mathbf{a}}_{i}+\beta \hat{\mathbf{a}}_{j} \\
& \hat{\mathbf{e}}_{j}=-\beta \hat{\mathbf{a}}_{i}+\alpha \hat{\mathbf{a}}_{j},
\end{aligned}
$$

where $\alpha^{2}+\beta^{2}=1$. The NCS for the two eigenvalues will add to the signature at the same location on the $\lambda$ axis, namely, $\lambda_{i}=\lambda_{j}$, because the unit step functions will fuse into a single unit step function, whose height is

$$
\left(\hat{d}_{i}\right)^{2}+\left(\hat{d}_{j}\right)^{2}
$$

By the Pythagorean Theorem, the quantity $\left(\hat{d}_{i}\right)^{2}+\left(\hat{d}_{j}\right)^{2}$ represents the squared length of the hypotenuse, namely, the component of $\hat{\mathbf{v}}$ in the plane defined by the eigenvectors. This quantity is a constant, independent of which coordinate system was used to calculate it. This can be proven by calculating $\left(\hat{d}_{i}\right)^{2}+\left(\hat{d}_{j}\right)^{2}$ in terms of $\mathbf{E}$ and A, and showing them to be equal.

$$
\begin{aligned}
& \left(\hat{\mathbf{v}} \cdot \hat{\mathbf{e}}_{i}\right)^{2}+\left(\hat{\mathbf{v}} \cdot \hat{\mathbf{e}}_{j}\right)^{2}= \\
& \left(\alpha \hat{\mathbf{v}} \cdot \hat{\mathbf{a}}_{i}+\beta \hat{\mathbf{v}} \cdot \hat{\mathbf{a}}_{j}\right)^{2}+\left(-\beta \hat{\mathbf{v}} \cdot \hat{\mathbf{a}}_{i}+\alpha \hat{\mathbf{v}} \cdot \hat{\mathbf{a}}_{j}\right)^{2}= \\
& \left(\alpha^{2}+\beta^{2}\right)\left[\left(\hat{\mathbf{v}} \cdot \hat{\mathbf{a}}_{i}\right)^{2}+\left(\hat{\mathbf{v}} \cdot \hat{\mathbf{a}}_{j}\right)^{2}\right]+(2 \alpha \beta-2 \alpha \beta)\left[\left(\hat{\mathbf{v}} \cdot \hat{\mathbf{a}}_{i}\right)\left(\hat{\mathbf{v}} \cdot \hat{\mathbf{a}}_{j}\right)\right]= \\
& \left(\hat{\mathbf{v}} \cdot \hat{\mathbf{a}}_{i}\right)^{2}+\left(\hat{\mathbf{v}} \cdot \hat{\mathbf{a}}_{j}\right)^{2}
\end{aligned}
$$

Given two medial node pairs in different images, a signature metric of similarity from node 1 to node 2 can now be defined as

$$
M_{1,2}=\int_{0}^{1}\left[s_{1}(\lambda)-s_{2}(\lambda)\right]^{2} d \lambda,
$$

where $0 \leq M_{1,2} \leq 1$. A value of 0 indicates identical signatures. The integral in Equation 10 can be computed efficiently as the area of a series of rectangles by first sorting the union of the two sets of eigenvalues from least to greatest, assigning a new index from $0 \leq h<2 N$ to each eigenvalue. The signature metric $M_{1,2}$ is then calculated by going through this set of $2 N$ eigenvalues

$$
M_{1,2}=\sum_{h=1}^{2 N} \kappa\left(\hat{d}_{h}\right)^{2}\left(\lambda_{h}-\lambda_{h-1}\right),
$$

where $\kappa=1$ for eigenvalues from node 1 and $\kappa=-1$ for eigenvalues from node 2 . This yields the same result as the continuous integral in Equation 10. 
Cross-image node pairs are said to correspond if (1) the unary metrics for the individual nodes are within tolerance, (2) the distance between nodes for each pair is sufficiently similar, and (3) both signature metrics (from node 1 to node 2 and vice versa) are also within tolerance.

\subsection{Medial Node Triplets}

Correspondences between cross-image node pairs may not be specific enough. Therefore, we introduce correspondences between medial nodes triplets. Greater specificity of medial node correspondences can be achieved if the geometric configuration between three nodes is considered. The grouping of three medial nodes into a medial node triplet or 3-clique is shown in Fig. 5.
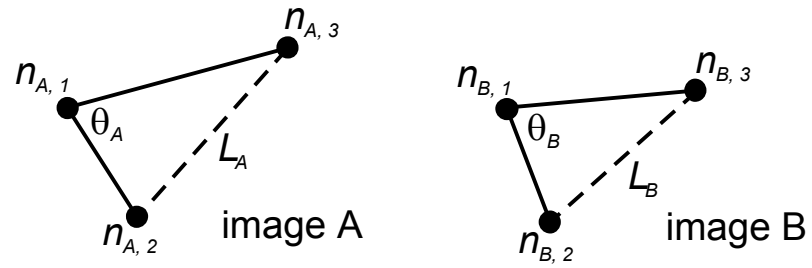

Fig. 5. Triplets from two images. Each vertex is a medial node, where $n_{\alpha, j}$ denotes the $j_{t h}$ node of the triplet in image $\alpha$.

Correspondences between triplets are implied from corresponding node pairs if the difference in angles between them is within a pre-determined threshold. For example, in Fig. 5, suppose node pairs $\left(n_{A, 1}, n_{A, 2}\right)$ and $\left(n_{A, 1}, n_{A, 3}\right)$ make up a triplet in image A and node pairs $\left(n_{B, 1}, n_{B, 2}\right)$ and $\left(n_{B, 1}, n_{B, 3}\right)$ make up a triplet in image B. These two triplets correspond if $\left|\theta_{A}-\theta_{B}\right|<\tau$, where $\tau$ is a pre-determined tolerance. Alternatively, distances $L_{A}$ and $L_{B}$ may be used.

\section{Results}

Three sets of data were used to demonstrate the ability of the unary and signature metrics to identify node correspondences. We first used an 8-bit, three-dimensional computer generated dataset consisting of two concentric ellipsoids. Voxels within the inner ellipsoid had an intensity of 255 and voxels between the inner and outer ellipsoid had an intensity of 128 . 

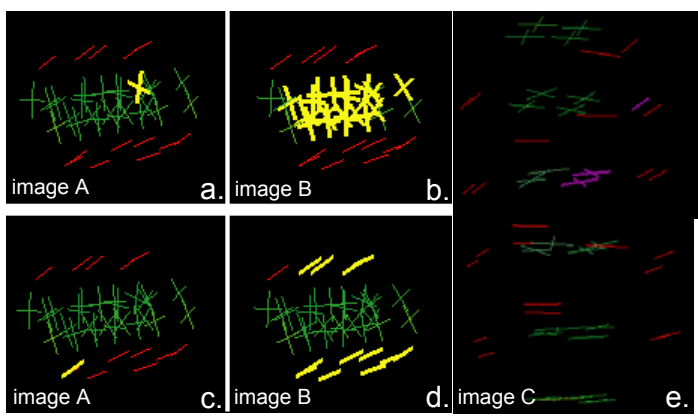
image $D$

Fig. 6. a) A node within the inner ellipsoid of image $\mathrm{A}$ is selected. b) The corresponding nodes in image B are highlighted. c) A node between the ellipsoids of image A is selected. d) The corresponding nodes in image B are highlighted. e) A triplet is selected in image C. f) The corresponding triplet is highlighted in image $\mathrm{D}$. g) The lambda triangle designates dimensionality as a function of eigenvalues $\lambda_{1}$ and $\lambda_{2}$. Arbitrary thresholds placed on $\lambda_{1}$ and $\lambda_{2}(\rho=1 / 5$ and $\gamma=1 / 3$ ) divide the triangle into 3 compartments, which permits dimensionality to be color-coded for visualization purposes.
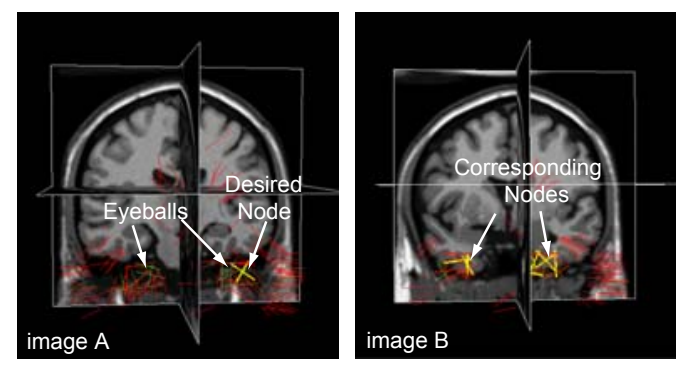

Fig. 7. MRI brain data shows that selecting a node in an eyeball of image A, will highlight corresponding nodes in both eyeballs of image B.

Correspondences between individual nodes were found between these two images using the unary metric. Figures 6 a through $6 \mathrm{~d}$ show slab-like medial nodes (core atoms that form between the ellipsoids) as red lines and cylindrical medial nodes (core atoms that form across the inner ellipsoid) as green crosshairs, following the colorcoding shown in the lambda triangle in Fig. 6g. In Fig. 6a, a node within the inner ellipsoid is selected (yellow crosshair) and the corresponding nodes are shown in Fig. 6b. In Fig. 6c, a node between the ellipsoids is selected (yellow line) and the corresponding nodes are shown in Fig. 6d. As expected only similar nodes are highlighted but there exists more than one corresponding node. This demonstrates the utility of the unary metric as well as the need for further specificity using node cliques.

The signature metric was demonstrated on two concentric ellipsoids, the second ellipsoid rotated by $90^{\circ}$. Figure 6e shows a selected node triplet (purple lines) and Fig. 6f shows the only properly corresponding node triplet. Mentally rotating the image superimposes the corresponding nodes. 
The unary metric was applied to simulated MRI brain images (See Fig. 7). Image $A$ and image $B$ were randomly generated by the MRI simulator at BrainWeb ${ }^{[13]}$. Image $\mathrm{B}$ was also non-rigidly deformed. The eyeballs are easily identifiable, because their roughly spherical nature is detected as a cylinder. Cylindrical nodes were found in both the eyeballs. Figure 7 shows that a node in one eyeball of image A was selected and corresponding nodes are highlighted in both eyeballs in image B. We are presently applying the signature metric to such image data.

\section{Discussion}

We have developed a novel method to automatically identify correspondences between medial based image features. The founding metrics were demonstrated to be rotationally invariant and able to locate basic shapes. It is envisioned that this system will become the basis for a means to generate medial node models automatically from a training set, to capture shape variability and locate, as well as measure, objects in a set of images containing similar objects. Useful classification of objects could be achieved based on these correspondences. Potential applications include time series, cross-subject, and multi-modal image analysis.

\section{References}

1. Dawant, B.M. Non-Rigid Registration of Medical Images: Purpose and Methods, a Short Survey. in International Symposium on Biomedical Imaging. 2002. Washington D.C.: IEEE.

2. C R Maurer, J. and J.M. Fitzpatrick, A Review of Medical Image Registration, in Interactive Image-Guided Neurosurgery, R.J. Maciunas, Editor. 1993, American Association of Neurological Surgeons: Park Ridge, IL. p. 17-44.

3. Maintz, J.B.A. and M.A. Vergever, A Survey of Medial Image Registration. Medical Image Analysis, 1998. 2(1): p. 1-37.

4. Grimson, W.E.L., Object Recognition by Computer. 1990, Cambridge, MA: MIT Press.

5. Stetten, G., Automated Identification and Measurement of Cardiac Anatomy Via Analysis of Medial Primitives, in Dept. Biomedical Engineering. 1999, University of North Carolina, Chapel Hill.

6. Stetten, G. and S.M. Pizer, Medial-Node Models to Identify and Measure Objects in RealTime 3-D Echocardiography. IEEE Transactions on Medical Imaging, 1999. 18(10): p. 1025-1034.

7. Blum, H. and R.N. Nagel, Shape description using weighted symmetric axis features. Pattern Recognition, 1978. 10: p. 167-180.

8. Pizer, S.M. and C.A. Burbeck. Cores as the basis for object vision in medical images. in Medical Imaging '94: Image Perception, SPIE. 1994.

9. Furst, J.D. and S.M. Pizer. Marching optimal-parameter ridges: An algorithm to extract shape loci in 3D images. in Medical Image Computing and Computer-Assisted Intervention. 1998. Cambridge, MA: Springer.

10. Burbeck, C.A. and S.M. Pizer, Object representation by cores: Identifying and representing primitive spatial regions. Vision Research, 1995. 35(13): p. 1917-1930. 
11. Young, R.A., The Gaussian derivative theory of spatial vision: Analysis of cortical cell receptive field line-weighting profiles. General Motors Research Publication, 1985. GMR4920.

12. Tamburo, R., Gradient-Oriented Boundary Profiles for Shape Analysis Using Medial Features, in Bioengineering. 2002, University of Pittsburgh: Pittsburgh.

13.Kwan, R.K., A.C. Evans, and G.B. Pike, MRI Simulation-Based Evaluation of ImageProcessing and Classification Methods. Transactions on Medial Imaging, 1999. 18(11): p. 1085-1097. 